\title{
summary
}

\section{Clear dose response for pain relief with aspirin even in single dose studies}

Edwards JE, Oldman AD, Smith LA, Carroll D, Wiffen PJ, McQuay HJ, Moore A. Oral aspirin in postoperative pain: a quantitative systematic review. Pain 1999; 81:289-297

Objective A systematic review of the analgesic efficacy and adverse effects of single-dose aspirin compared with placebo in post-operative pain.

Data sources Medline 1966-1998, Embase 1980-1998, Cochrane Library Issue 1, 1998, Oxford Pain Relief Database 1950-1994, and manual search of the reference lists of retrieved papers using a detailed search strategy.

Study selection Only full journal publication of double-blind studies with randomly allocated adult patients receiving postoperative oral administration for treatment of moderate to severe pain baseline pain (equates to $>30 \mathrm{~mm}$ on a visual analogue scale, VAS), using acceptable pain measures. [Five-point pain relief scale with standard wording, a four-point pain intensity scale, a VAS for pain relief or intensity, or total pain relief (TOTPAR) or summed pain intensity difference (SPID) or their visual analogues.] Trials using aspirin in combination with other drugs, cross-over design as a single dataset, number of patients per treatment group $<10$, pain relief data collected after intake of additional analgesics, galenic formulations which may significantly alter absorption kinetics and baseline pain less than moderate to severe pain (VAS $<30$ $\mathrm{mm}$ ) were excluded. Abstracts and review articles were not sought. Language other than English was not a restriction. Independent quality scores were made.

Results Of the 175 publications identified, six were unobtainable from the British Library; of the remainder, 69 papers representing 72 trials met all the inclusion criteria, generating 88 aspirin versus placebo comparisons. $68 \%$ were in a dental setting. Single-dose aspirin showed significant benefit over placebo for 600/650 mg, $1000 \mathrm{mg}$ and $1200 \mathrm{mg}$ doses. There were insufficient data for 300/325mg and 900mg dosage. Sixty of the trials reported on adverse effects, giving an overall number needed to harm (NNH) for the 600/625 mg dose of 44 (95\% CI 23-345), with a NNH of 28 (95\% CI 19-52) for drowsiness and 38 (95\% CI 22174) for gastric irritation.

\begin{tabular}{lccccc}
\hline $\begin{array}{l}\text { Aspirin } \\
\text { dose } \\
(\mathrm{mg})\end{array}$ & $\begin{array}{c}\text { Number of } \\
\text { comparisons }\end{array}$ & \multicolumn{2}{c}{$\begin{array}{c}\text { Patients with at least } \\
50 \% \text { pain relief } \\
\text { Aspirin }\end{array}$} & $\begin{array}{c}\text { Risk ratio } \\
(95 \% \mathrm{Cl})\end{array}$ & $\begin{array}{c}\text { NNT } \\
(95 \% \mathrm{Cl})\end{array}$ \\
\hline 500 & 3 & $45 / 135$ & $32 / 115$ & 1.2 & $\mathrm{NC}^{*}$ \\
$600 / 625$ & 68 & $960 / 2499$ & $404 / 2562$ & $\begin{array}{c}(0.8-1.8) \\
2.0\end{array}$ & 4.4 \\
& & & & $(1.8-2.2)$ & $(4.0-4.9)$ \\
1000 & 5 & $153 / 357$ & $64 / 359$ & 2.2 & 4.0 \\
& 5 & $85 / 140$ & $27 / 139$ & $\begin{array}{c}(1.4-3.4) \\
(3.3\end{array}$ & $\begin{array}{c}(3.2-5.4) \\
1200\end{array}$ \\
& 5 & & & $(1.8-6.3)$ & $(1.9-3.2)$ \\
\hline
\end{tabular}

* Not calculated, as it was not significant

Conclusion There is a clear dose response for pain relief with aspirin even in these single-dose studies. Adverse effects of drowsiness and gastric irritation were also evident even though they were single-dose studies.

Supported by grants from NHSR\&D Health Technology Evaluation Programme (\#93/31/4 and \#94/11/4), European Union Biomed 2 BMH4 CT950172, Smith Kline Beecham Consumer Health Care, The Biotechnology and Biological Sciences Research Council, and Pain Research Funds.

Address for reprints: Dr A Moore, Pain Research and Nuffield Department of Anaesthetics, University of Oxford, Oxford Radcliffe NHS Trust, The Churchill, Headington, Oxford OX3 7LJ, UK

\section{Commentary}

Aspirin is one of the oldest peroral analgesics currently in use. It has traditionally been considered the 'golden standard' with which most other non-steroidal anti-inflammatory drugs have been compared. At present no other peroral analgesic has a longer history of widespread clinical use and empirical efficacy. Thus, it may come as no surprise that the authors claim the superiority of aspirin over placebo. For the dental clinician it must be reassuring that this conclusion comes from a meta-analysis with $68 \%$ of the trials originating from unspecified types of dental surgery.
The authors claim a clear doseresponse for the pain relieving effect of aspirin, that type of pain model had no influence on the results and stress the similar pain relieving effect of aspirin and paracetamol on a weight to weight basis. The authors' emphasis on the similarities of the analgesic effect of paracetamol and aspirin may mislead colleagues into the believing that aspirin and paracetamol have identical pharmacodynamic effects in dentistry.

The most interesting contribution from this paper is the information regarding adverse effects. It shows convincingly that pooled data from single dose studies can be a very good predictor of adverse effects after chronic use at least for aspirin. It is interesting that drowsiness is seen more frequently after dental surgery than after other procedures, although postoperative stress after dental surgery using only local anaesthetics might be a major contributing factor to this observation.

This paper does not provide any new information on how to put aspirin in therapeutic perspective for the dental clinician, but merits as background for improving clinical trial methodology.

Lasse A Skoglund

Section of Dental Pharmacology and Pharmacotherapy, University of Oslo, Norway 